\title{
Zur Stellung der Eigennamen im Wortschatz
}

\author{
Rainer Wimmer
}

\section{Einleitung}

Die in der Linguistik allgemein (keineswegs nur in der germanistischen Linguistik) bis heute angenommene, weithin akzeptierte und gelehrte Dichotomie zwischen Name (Eigenname $=$ EN) und Wort hat eine sehr alte, bis in die Antike zurückreichende Tradition und wird bis heute gestützt durch die in vielen Sprachgesellschaften verbreitete und intuitiv für plausibel gehaltene Vorstellung, daß es zwei grundverschiedene Dinge sind, eine Person oder einen Gegenstand mit einem Eigennamen (EN) zu benennen und fortan zu bezeichnen oder aber eine bestimmte Person bzw. einen bestimmten Gegenstand mit einem Wort, also einem Appellativum (dieser Mann, mein Onkel) zu bezeichnen. Eigenname und Wort gelten als sprachlich grundverschieden, was oft auch so ausgedrückt wird, daß Eigennamen gar nicht zum Wortschatz einer Einzelsprache gehören, sondern übereinzelsprachliche Qualität haben (Wird die Stadt London nicht in allen Sprachen mit London bezeichnet?) und deshalb auch mit Recht nicht in einem Sprachwörterbuch des Deutschen, Italienischen, Englischen, Chinesischen usw. aufgeführt werden, sondern allenfalls (nämlich bei entsprechender "Bedeutung" der bezeichneten Gegenstände) in Enzyklopädien der Sachen, der Personen, Länder, Ereignisse usw. Von daher nährt sich die Meinung, EN hätten bezüglich des Wortschatzes einer Einzelsprache eine Randstellung, sie hätten nur peripher mit der Bedeutungsstruktur eines Einzelsprachwortschatzes zu tun. Diese Meinung kann allerdings nur dann substantiiert werden, wenn man unter dem Wortschatz einer Sprache das Langue-Abstraktum einer Gesamtsprache wie des Deutschen versteht, d.h. den deutschen Wortschatz. Aber was ist das? Kennt oder beherrscht ihn ein einzelner Deutscher? Antwort: Nein. Die "Rückläufige Wortliste" (Brückner/Sauter 
1984) über gegenwartssprachliche Texte des Deutschen, die im Institut für deutsche Sprache maschinenlesbar für lexikographische und grammatische Untersuchungen genutzt werden, enthielt schon vor Jahren mehr als 150.000 Einträge (Wörter); Lexikographen sprechen sonst von 450.000 bis 500.000 Wörtern der deutschen Gegenwartssprache; also: Wenn der aktiv beherrschte Wortschatz eines einzelnen Sprechers des Deutschen fünf- bis zehntausend Wörter umfaßt (wie meistens angenommen wird), dann kann ein einzelner Sprecher nicht den deutschen Wortschatz insgesamt beherrschen; aber er beherrscht die für ihn wichtigen Eigennamen. Ich ziehe aus solchen (groben) Zahlen den Schluß, daß man zwischen dem Wortschatz einer Gesamtsprache (wie dem Deutschen) und dem von einzelnen Sprechern beherrschten Wortschatz strikt unterscheiden muß. Der Wortschatz eines einzelnen ist in Wortschätzen von Einzelsprachen enthalten; jeder Wortschatz einer Einzelsprache geht über den Wortschatz eines einzelnen Sprechers hinaus. Aber: Einzelne Sprecher partizipieren auch (mehr oder weniger) an den Wortschätzen verschiedener Sprachen. Lehnwörter bereichern nicht nur das Wörterbuch einer Langue als Gesamtsprache, sondern auch den Wortschatz jedes einzelnen Sprachteilnehmers, oft ohne daß diesem der übereinzelsprachliche Gebrauch von Wörtern und Wendungen bewußt ist. Beim Gebrauch von Fachsprachen und anderen terminologisch fixierten Ausdrucksformen gehen wir mit Selbstverständlichkeit davon aus, daß wir es in vielen Fällen mit einem übereinzelsprachlichen Wortschatz, d.h. mit Internationalismen zu tun haben (vgl. Braun/Schaeder/Volmert 1990); der "interlinguale" Charakter von Ausdrücken wie Demokratie, Politik, Liberalismus, Sozialismus - um einige Beispiele aus dem Bereich des politischen Sprachgebrauchs zu nennen - wird heute von vielen Sprachteilnehmergruppen akzeptiert, obwohl - und das scheint mir sehr bemerkenswert die Meinungen darüber, was mit Demokratie usw. jeweils bezeichnet wird, und damit die Gebrauchsweisen bzw. Bedeutungen der Ausdrücke weit auseinandergehen und bei den Sprechern sogar ein Bewußtsein für die zum Teil extremen Bedeutungsdivergenzen vorhanden ist.

Ich ziehe aus diesen allgemeinen Vorbemerkungen zu Wortschatzfragen folgende Konzequenzen:

- Die Themafrage nach der Stellung der EN in Wortschatz muß vor allem unter der Perspektive einer notwendigen Differenzierung des Wortschatzbegriffs betrachtet werden.

- Der Begriff "Langue-Wortschatz einer Einzelsprache" beinhaltet eine sehr viel weitergehende Abstraktion von der Individualsprache (nicht: 
"Privatsprache") und Individual-Sprachkompetenz des einzelnen Sprechers als etwa der Begriff der "Einzelsprachensyntax", und dies insofern, als spezifische Gebrauchsweisen bzw. Bedeutungen von Wortschatzelementen (z. B. Termini, EN) im Rahmen der Sprachkompetenz einzelner Sprecher einen weitergehend interlingualen Charakter haben als spezifische Syntaxregeln. Die schwierige Frage, inwieweit man hier unter Bezug auf das Konzept einer Universalgrammatik von einer Parallele zwischen Syntax und (Wortschatz-)Semantik sprechen könnte - was ich persönlich nicht glaube - lasse ich außer Betracht.

- Die Wortschatzkompetenz des einzelnen Sprachteilnehmers ist sehr viel weniger einzelsprachlich ausgeprägt, als es das Sprachbewußtsein der Sprecher, vor allem das Nationalsprachenbewußtsein der Deutschen seit dem 19. Jahrhundert, und die auf das Einzelsprachsystem orientierte linguistisch-strukturalistische Theoriebildung wahrhaben wollen.

\section{Thesen}

Die Vorbemerkungen deuten bereits eine bestimmte These zur EN-Semantik an; und sie sind deshalb auch nicht als ein Referat gängiger linguistischer Meinungen zu Wortschatzfragen aufzufassen. Entgegen verbreiteter Auffassungen in der Linguistik und in der Alltagsreflexion über Sprachfragen möchte ich die These vertreten, daß

(a) die unter semantischen Gesichtspunkten angenommene strikte Trennung zwischen Appellativa einerseits und EN andererseits im Wortschatz einer Sprache nicht adäquat und nicht sehr fruchtbar ist, wenn man die sprachliche Kommunikation unter Verstehensgesichtspunkten analysieren, beschreiben und erklären will,

(b) die verbreitete Auffassung, EN seien "deskriptiv leer", hätten im Gebrauch lediglich eine Bezeichnungsfunktion und seien deswegen möglicherweise sogar semantisch "defizitär", unter den angedeuteten kommunikationsanalytischen Gesichtspunkten nicht sinnvoll erscheint,

(c) die Rolle, die EN-Bedeutungen im (individualsprachlichen) Wortschatz spielen, linguistisch meistens unterschätzt wird. Möglicherweise kann der Aufbau von EN-Bedeutungen im Laufe von Kommunikationsgeschichten sogar als prototypisch für den Aufbau und die Weiterentwicklung von Bedeutungen im substantivisch-appellativischen Wortschatz angesehen werden. 
Diese aus mehreren Teilen bestehende These kann im folgenden natürlich nur relativ knapp und grob erläutert, vielleicht auch plausibel gemacht werden. Soweit die These weit verbreitete und grundsätzliche Positionen in der germanistischen Lexikologie und Lexikographie zu relativieren versucht, wären ausführliche, ja langwierige Erörterungen, auch mit wissenschaftsgeschichtlicher Perspektive, erforderlich, wollte man die Hoffnung haben, die Begründetheit der These für mehr als einen kleinen Kreis von ohnehin unkonventionell Denkenden zu erweisen. Ich vermute, daß merkmalsemantisch orientierte Lexikologen und Lexikographen (auch wegen der Konsequenzen für die praktische Wörterbucharbeit) die These eher für gewagt bis abwegig halten, daß linguistische Namenforscher die Position diskutabel finden könnten und daß sprachphilosophisch orientierte Semantiker vielleicht gar nichts Neues an all dem finden, eher noch die mangelnde Konsequenz bemängeln müßten. Es ist aus linguistischer Sicht bemerkenswert, daß die Eigennamensemantik in der wahrheitswert-funktional orientierten philosophischen Semantik immer schon eine wichtige, wenn nicht gar zentrale und prototypische Rolle gespielt hat. Seit über hundert Jahren dominiert die Kontroverse um die Eigennamentheorie wichtige Bereiche der philosophischen Semantik (vgl. Wolf 1985, dort auch die Einleitung, S. 9-41), und man kann sich sehr wohl fragen, ob darin nicht doch auch ein Indiz dafür gesehen werden darf, daß die Struktur der EN-Bedeutung nicht völlig peripher für den semantischen Aufbau des (natürlichsprachlichen) Wortschatzes ist.

\section{3. "Randphänomen" Eigenname}

Peter von Polenz hat in seinen früheren onomastischen Arbeiten von der "Ausschaltung der Wortschatzstruktur beim Namen" (v. Polenz 1960/61: 9) gesprochen, dies allerdings bezogen auf den Namengebrauch nach dem erfolgten Namengebungsakt. In der Tat ist das Bild vom "Ausschalten" der Wortschatzstruktur ein treffendes Bild für die im Nachhinein oft schlagartig erscheinende Veränderung der Bezeichnungsmotivik und -funktion von Ausdrücken im Namengebungsakt bzw. "Referenzfixierungsakt" (vgl. Wimmer 1973; 1979): Wer in einem Namengebungsakt gut motiviert mit der Berufsbezeichnung Müller (als einem Familiennamen) bedacht wird, ist möglicherweise bereits unmittelbar nach der Namengebung nicht mehr als Müller tätig (d. h. die semantischen Merkmale der Berufsbezeichnung Müller treffen nicht mehr auf ihn zu), ja selbst im Augenblick der Namengebung müssen die semantischen Merkmale nicht unbedingt auf den zukünftigen Namenträger zutreffen; es 
genügt, wenn die appellativischen Merkmale als Motive bei den Namengebern fungieren. Freilich gibt eine solche Beschreibung ("Ausschaltung der Wortschatzstruktur") nicht die tatsächlichen sprachhistorischen oder kommunikationshistorischen Verhältnisse wieder; der Weg vom Appellativum zum EN ist oft lang; der umgekehrte Weg übrigens auch. Das Phänomen des Wechsels zwischen bedeutungsvollem Appellativum und einem Sprachzeichen, das als EN im wesentlichen nur mit Bezeichnungsfunktion gebraucht wird, läßt sich aber auch aktuell beobachten, beispielsweise bei Spitznamen, Beinamen oder Übernamen. Werner Kany hat am Beispiel von "inoffiziellen Personennamen" gezeigt, wie Sprecher mit dem Wechsel von mehr prädikativ-deskriptivem Wortgebrauch zu rein referierendem EN-Gebrauch (und umgekehrt) spielen (vgl. Kany 1992). Mit der Bezeichnung Osram für einen Fußballtrainer kann nur der richtig umgehen, der weiß, daß der Kopf dieses Trainers in Erregungszuständen wie eine Glühbirne erscheint (Beispiel aus der Belegsammlung von Kany).

Es ist ein Topos in der Onomastik und auch in der allgemeinlinguistischen Beschreibung von EN (vgl. z. B. Ziff 1960: 86), daß mit EN bezeichnet wird, ohne daß mit einer solchen Bezeichnung bestimmte Prädikationen über den bezeichneten Gegenstand verbunden werden; d.h. EN werden als rein referentiell, als nicht-deskriptiv angesehen. Jeder Sprecher verbindet mit dem Gebrauch von Ausdrïcken wie Tisch, Stuhl, Hocker; Rappe, Schimmel (derartige Ausdrücke werden vorzugsweise in der einschlägigen Handbuchliteratur behandelt) bestimmte semantische Merkmale ('zum Sitzen', 'vier Beine', 'mit Lehne'; 'schwarz', 'weiß'); das gehört zur Sprachkompetenz der Sprecher. Anders bei EN: Die Möglichkeit, mit einem EN wie Apollo sowohl auf einen griechischen Gott wie auch auf ein amerikanisches Raumfahrtprogramm wie auch auf einen Hund (Haustier; die Reihe ließe sich fortsetzen) Bezug zu nehmen, soll gerade deshalb gegeben sein, weil EN nicht mit bestimmten semantischen Merkmalen verknüpft sind. Die Beobachtung der sog. Bedeutungslosigkeit von EN wird oft mit folgenden Hinweisen gestützt:

- EN werden meistens nicht übersetzt.

- EN werden normalerweise nicht in einsprachige Wörterbücher aufgenommen. Es gibt in vielen Sprachen eine relativ strikte Trennung zwischen Wörterbüchern einerseits und Namenlexika bzw. Enzyklopädien andererseits.

- Das Lernen von EN-Verwendungen wird normalerweise nicht als Teil des Sprachenlernens beim Zweitsprachenerwerb angesehen. 
- EN nicht zu kennen, gilt normalerweise nicht als Mangel in der Sprachkompetenz.

Daß EN als ein "Phänomen" am Rande des Wortschatzes einer Sprache angesehen werden, hängt mit all diesen angenommenen Eigenschaften von Namen zusammen. Aber kann man nicht mit meiner oben formulierten These fragen, ob EN in der individuellen Sprachkompetenz eines Sprechers nicht eine ganz andere Rolle spielen? Man mag das Experiment machen (letztlich wird man es keine zwei Stunden am Tag durchhalten), zu kommunizieren, ohne die vertrauten EN aus dem eigenen Lebenszusammenhang zu gebrauchen, ohne sie auch nur zu erwähnen. Wäre das möglich? Sind nicht die EN eigentlich das Kernstück unserer individuellen sprachlichen Orientierung in unserem alltäglichen Umfeld?

\section{Der Eigenname als der Prototyp im Wortschatz}

Am EN hängt sich die Bedeutungsentwicklung beim Erstspracherwerb auf. Man kann darüber spekulieren, ob die sog. Einwortsätze, von denen die Spracherwerbstheoretiker sagen, sie seien der Anfang des Grammatik- und damit auch Bedeutungserwerbs der Kinder, EN sind. Aber warum eigentlich nicht? Eigennamen (Personennamen, Ortsnamen usw.) stellen den Kern des sprachlichen und "mentalen" Wissens dar, mit dem wir uns in unserer Umwelt orientieren. Wenn man "Lexikon" systematisch doppeldeutig versteht, wie es Linguisten meistens tun, nämlich als das, was einerseits im Kopf von Sprechern als Wortschatz "gespeichert" ist und was andererseits als Beschreibung dessen in Wörterbüchern aufgeschrieben ist, so kann man sich fragen, warum diese beiden Lexika so sehr divergieren sollen? Warum sollen in den aufgeschriebenen und publizierten Wörterbüchern für eine Sprache nicht auch die EN verzeichnet werden, die in unseren Köpfen eine so große Rolle spielen? Meine These ist, daß die Linguistik gut daran täte, die strikte (theoretische) Trennung zwischen EN einerseits und Appellativa andererseits aufzugeben. Der Universal-Duden nimmt ein Wort wie Isis (ägyptische Göttin) als Lemma auf. Warum nicht auch Iphigenie, die in vielen deutschen Köpfen eine größere Rolle spielt? Die Eigennamen in unserer engeren und weiteren Alltagsumgebung sind die Kristallisationspunkte für den Aufbau von Wortbedeutungen. Daß Schimmel weiß sind und Rappen schwarz, ist peripher für unser Sprachhandeln. 